\title{
Study of SEM images obtained with an electron energy and take-off angle $(E-\theta)$ selective detector
}

Takeshi Otsuka ${ }^{1}$, Motohiro Nakamura ${ }^{1}$, Ken-ichi Yamashita ${ }^{1}$, Masaya Hara ${ }^{1}$, Felix Timischl ${ }^{2}$, Kazuhiro Honda ${ }^{1}$, Masato Kudo ${ }^{2}$ and Shin-ichi Kitamura ${ }^{1}$

1. JEOL Ltd., 1-2 Musashino, 3-Chome, Akishima, Tokyo, 196-8558, Japan

2. JEOL Technics Ltd., 6-38 Musashino 2-chome, Akishima, Tokyo, 196-0021, Japan

Scanning electron microscopes (SEMs) are usually equipped with different types of electron detectors. This allows acquisition of images showing diverse contrast, which is caused by the range of detectable energy and take-off angle, respectively. However, the phenomenon is not fully understood yet. We have manufactured an $E$ - $\theta$ detector, which can detect electrons emitted from a sample with a selectable range of both energy and take-off angle [1].

Figure 1 shows a schematic diagram of the $E-\theta$ detector. The operation is as follows:

1. The aperture plate acts as a selector of take-off angle. The plate has two types of apertures; one for high angle range detection and one for low angle range detection.

2. Electron energy selection is carried out by applying voltages of opposite polarity to the inner and outer electrodes as shown in Figs. 1(a) and (b). The polarity of the applied voltages is reversed in the two cases of high angle and low angle range detection, so that the deflection direction of the electrons is reversed. Consequently, each detection element detects a different electron energy range.

3. We use a circular Si-photodiode (SiPD) as the electron detection device, which is separated into concentric elements. A high voltage is applied between the metal mesh and the detection elements to accelerate the incoming electrons.

Figure 2 shows a set of images from the same area of a solder sample with high and low angle ranges and high and low energy ranges. The high energy and high take-off angle image B shows composition contrast. The high energy and low take-off angle image D shows a clear shadow effect. Edge effect can be seen in the low energy images $\mathrm{A}$ and $\mathrm{C}$, however it is more pronounced in the low angle image $\mathrm{C}$ than in the high angle image A.

Figure 3 shows a set of images of spherical polystyrene particles with a diameter of $1 \mu \mathrm{m}$ taken at $10 \mathrm{kV}$ accelerating voltage. The low energy range images $\mathrm{A}$ and $\mathrm{C}$, and the high energy and high take-off angle range image $\mathrm{B}$ show charging effects, proving that different take-off angle and energy ranges contribute to contrast corruption by the charging mechanism. The high energy and low take-off angle range image $\mathrm{D}$ is not affected by charging.

These images show clear differences, which indicate the usefulness of the $E-\theta$ detector. We are now manufacturing a smaller $E-\theta$ detector for use at short working distances. In the presentation, we will show a series of high magnification images obtained with different ranges of energy and take-off angle.

References:

[1] T. Otsuka et al, Microscopy and Microanalysis (2014) p. 36. 


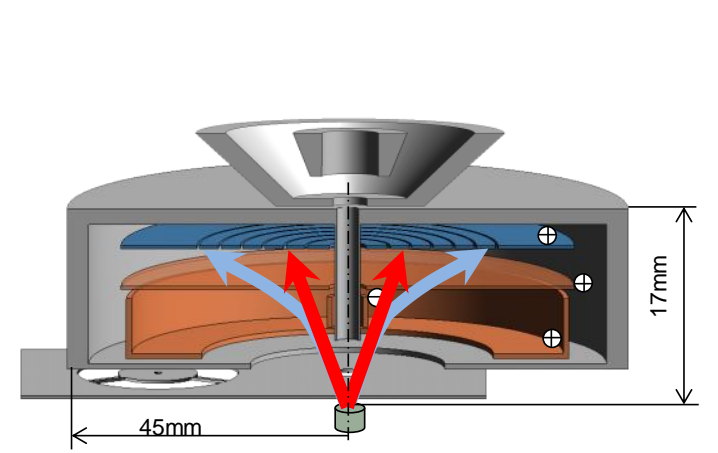

(a) High angle range detection setup

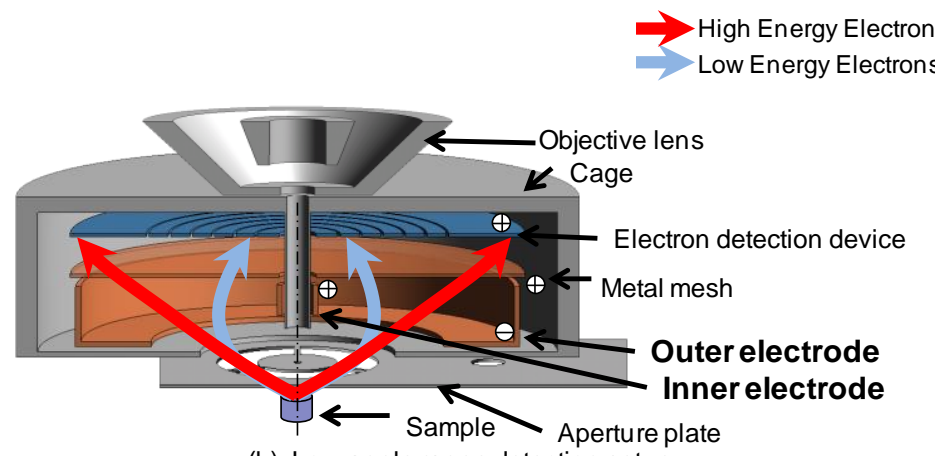

(b) Low angle range detection setup

Figure 1. Schematic diagram of the $E-\theta$ detector
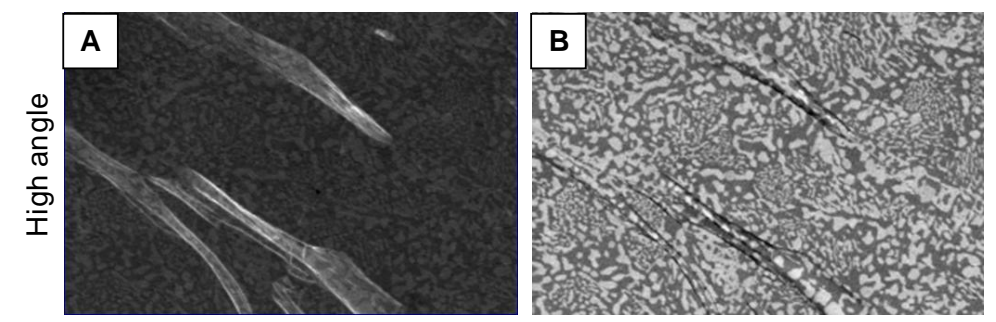

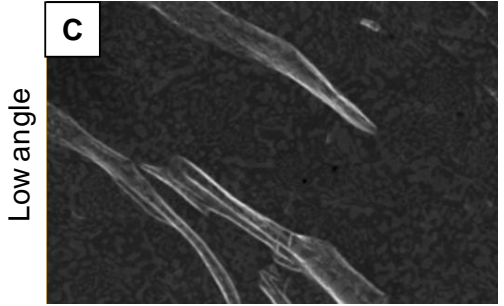

Low energy

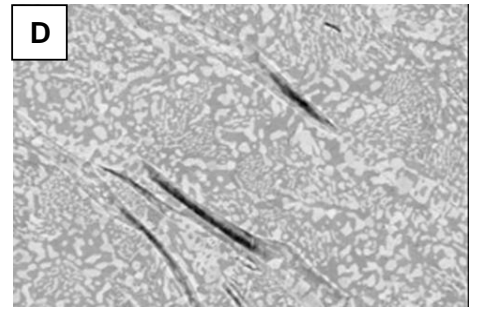

High energy

\section{$10 \mu \mathrm{m}$}

Figure 2. Topographic and composite contrast images of a solder sample taken at $10 \mathrm{kV}$.

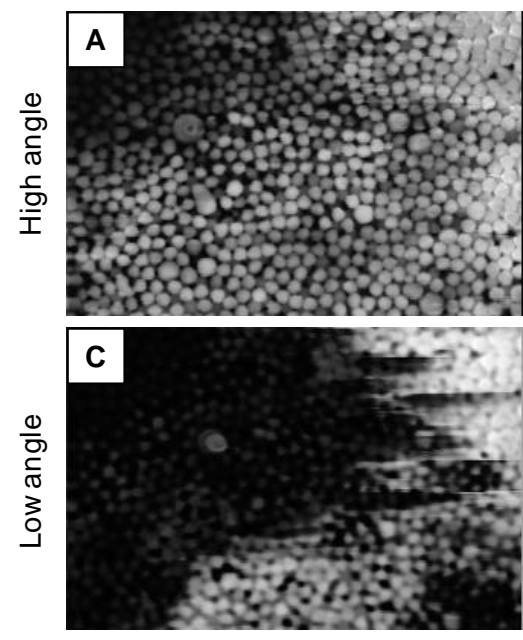

Low energy
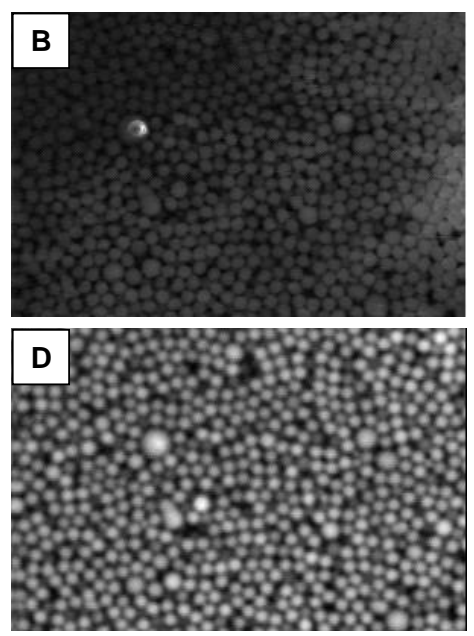

High energy
$10 \mu \mathrm{m}$

Figure 3. Charging contrast images of spherical polystyrene particles with diameter of $1 \mu \mathrm{m}$ taken at 10 $\mathrm{kV}$. 